\title{
Medical radiation dosimetry - theory of charged particle collision energy loss by Brain McParland
}

\author{
Richard James Crilly
}

Received: 20 July 2014 / Accepted: 3 September 2014 / Published online: 16 September 2014

(C) Springer-Verlag Berlin Heidelberg 2014

In the daily grind of clinical physics, we are often confronted with the need to make intelligent decisions on the accuracy of our computer-generated calculations. To do so, we fall back on a standard list of assumptions to justify our tack. If pressed, we review a plethora old papers and notes to justify our actions. This problem, of having a single comprehensive source, has been partially simplified with the publication of Medical Radiation Dosimetry-Theory of Charged Particle Collision Energy Loss by Brain McParland. In this book, Dr. McParland authors a detailed, clear development of charge particle energy deposition.

In the foreword, the author posits that for purposes of medical applications, the study of radiative losses is insignificant, though he does concede that this may not be true for high energy electrons. Given the current work done to improve dosimetric accuracy by fractions of a percent, I am not certain I entirely agree with this line of argument. Nonetheless, I agree on the primary importance of understanding the theory underlying charged particle energy loss.

The majority of the book is clearly meant for the physicist and has little to offer anyone without a clear understanding of basic quantum physics, electromagnetic theory, and higher order mathematics. A clear exception to this assertion would be is the extensive introduction which is a wonderful essay summarizing earlier models. It is in the form of a historical narrative that allows us to envision not only the series of incremental steps that lead to the modern theory but also the numerous miss-steps and near misses that are the hall mark of scientific advancement.
The actual development of physics theory starts with the second section that entails a review of quantum scattering theory. Thankfully, for this reviewer, the text does not assume the reader's last quantum class was a number of weeks ago and eases into the subject carefully outlining each assumption and approximation made along the way. This section lays the ground work for the second part of the book wherein the topics of elastic Coulomb scatter are covered in great detail from simple classical solutions to multiple elastic Coulomb scatter including Fermi-Eyges theory. Part three covers the development of collisional energy concepts including collisional stopping power, charged particle range, and the continuous slowing down approximation. This is done, as in previous sections, by starting with simpler models and folding in additional complexities. The final section develops the probability distributions for thin foil scattering and reviews the limitations of such models. Throughout the text, the assumptions and approximations that inherently limit the applicability of any theory are laid out in a clear and precise manner.

It would be dishonest to suggest that I have read this text of such a complex subject cover to cover. Instead, I have used it to enhance and reacquaint myself with those aspects of charged particle dosimetry I have an abiding interest in. Will I ever read this book cover to cover? No, but I plan to place it close by for easy access and reference.

Conflict of interest The author declares that he has no conflict of interest.
R. J. Crilly $(\bowtie)$

Oregon Health and Science University, Portland, OR, USA

e-mail: crilly@ohsu.edu 